\title{
Carbapenems in the treatment of intra-abdominal infection
}

\section{Joanna Lumb}

London, UK=jlumb@f2s.com

\section{Report from the 20th European Congress of Clinical Microbiology and Infectious Diseases}

\section{Vienna, Austria, 10-13 April 2010}

The changing pattern of resistance to antibacterial agents was a major theme at the 20th European Congress of Clinical Microbiology and Infectious Diseases. This article highlights presentations on the increasing threat from multidrugresistant Gram-negative pathogens, particularly extended-spectrum $\beta$-lactamase-producing Escherichia coli and Klebsiella pneumoniae, which are limiting the choice of drug therapy for serious infections. Surveillance data suggest that carbapenem antibiotics are appropriate choices for the empiric treatment of intra-abdominal infection.

\section{European increase in resistance}

A rapid increase in resistant Gram-negative pathogens was highlighted in a report from the European Antimicrobial Resistance Surveillance System, which collects data from 33 countries.

Roede and colleagues analyzed trends over the past 4 years in resistance of invasive Escherichia coli and Klebsiella pneumoniae infections and found increasing loss of effective antimicrobial therapy [1]. Fluoroquinolone resistance increased significantly over the study period in 19 countries. Resistance to third generation cephalosporins increased in 21 countries. Combined resistance (to third generation cephalosporins, fluoroquinolones and aminoglycosides) was the dominant threat.

For carbapenems, resistance in $K$. pneumoniae was still not seen in 21 out of 31 countries; however, seven countries reported resistance rates of $1-5 \%$ and rates were much higher in three countries (Cyprus, Israel and Greece).

\section{Intra-abdominal infection}

Further data presented at the European Congress of Clinical Microbiology and Infectious Diseases (ECCMID)show that the options for effective empirical therapy of intra-abdominal infection in Europe have reduced.

The Study for Monitoring Antimicrobial Resistance Trends (SMART) program has been monitoring the activity of antibiotics against aerobic Gram-negative intra-abdominal infections. Badal and colleagues (International Health Management Associates, USA) reported susceptibility levels of key intra-abdominal pathogens in Europe for 2008/9, based on samples from 46 laboratories in 14 countries [2]. Susceptibility to the following antibiotics was tested: ertapenem, amikacin, cefepime, cefoxitin, ceftazidime, cefriaxone, ciprofloxacin, imipenem, levofloxacin, ampicillin/sulbactam and piperacillin/tazobactam.

Only three antibiotics (imipenem, ertapenem and amikacin) had susceptibility levels of $90 \%$ and higher against $E$. coli, which accounted for around $50 \%$ of all pathogens. Only the two carbapenems - imipenem and ertapenem - had susceptibility levels of $90 \%$ or higher against $K$. pneumoniae (11\% of pathogens); and for just one other drug (amikacin) was susceptibility over $80 \%$. No drug achieved $80 \%$ susceptibility for Pseudomonas aeruginosa.

Extended-spectrum $\beta$-lactamase (ESBL)positive rates in intra-abdominal infections were nearly 12 and $22 \%$ for E. coli and K. pneumoniae, respectively, accounting for approximately $9 \%$ of pathogens.

The researchers concluded that unless ESBLpositive organisms have been ruled out by laboratory testing, the most effective agents appear to be imipenem, ertapenem or amikacin for empiric therapy of intra-abdominal infection in Europe.

Gram-negative bacterial resistance increases the burden on hospitals with respect to mortality, length of stay and costs. This is especially true in intensive care units (ICUs) where antibiotic resistance tends to be higher than non-ICU settings.

In another poster presentation, the SMART researchers compared susceptibility of Gramnegative pathogens from intra-abdominal infections in ICU and non-ICU settings in 2 years, 2008 and 2009. This study involved samples from 134 laboratories in 40 countries worldwide [3].

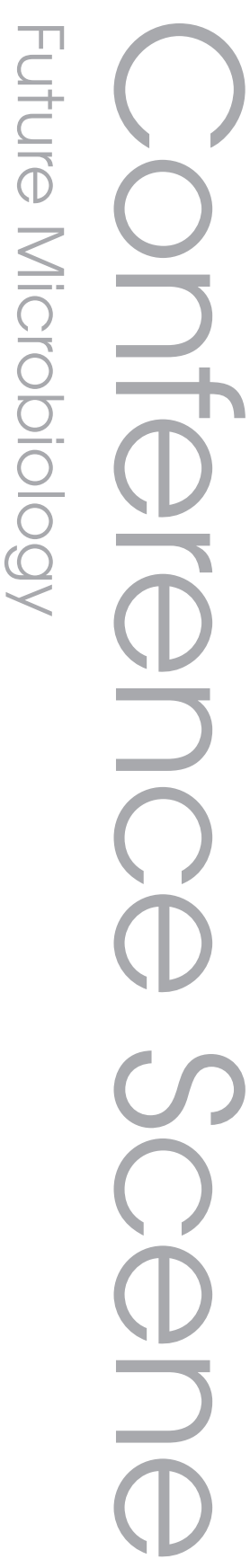

\section{future medicine $^{\text {posg }} \mathbf{s g}$}


As expected, the data suggest that intra-abdominal infection pathogens from ICU are often more resistant to commonly used drugs, although this did not apply to all pathogens. Ertapenem and amikacin demonstrated more consistent activity between ICU and non-ICU isolates than the other drugs evaluated and only ertapenem maintained susceptibility of $90 \%$ or higher in both ICU and non-ICU clinical settings against all species for which it has approved indications (which does not include Pseudomonas).

\section{Experience with ertapenem in one US hospital}

A common practice when a new broad-spectrum antibiotic is marketed is to limit its use in an attempt to minimize development of resistance. At one large US hospital, the opposite approach was taken when the carbapenem antibiotic ertapenem was marketed in 2002. Its use was encouraged as a way of preserving the activity of another carbapenem imipenem for treating serious pseudomonal infections in ICU patients.

There has been concern that use of ertapenem will negatively affect the activity of imipenem, but to date that has not been the case: a 6-year study shows that introduction of ertapenem has not affected the susceptibility of Pseudomonas aeruginosa (or other Gram-negative organisms) to imipenem [4].

The study was reported by Debra Goff, infectious disease pharmacist at the 1510-bed Ohio State University Medical Center. She explained that at the time ertapenem was approved in 2002, the hospital had noted an increase in ESBL-producing $K$. pneumoniae, from $4 \%$ in 2001 to $9 \%$ in 2002.

It was decided that ertapenem would be useful for treating ESBL-producing pathogens without needing to resort to imipenem. Ertapenem was therefore added to the hospital formulary in
2003 and its impact on bacterial susceptibility to imipenem has been monitored. From 2002 to 2007, the percentage of imipenem-susceptible Pseudomonas aeruginosa isolates remained stable at around $70 \%$ and susceptibility actually improved in 2008. Over the study period, overall use of carbapenem antibiotics increased; as expected, ertapenem was increasingly used as an alternative to ampicillin/sulbactam for infection in postoperative patients.

Notwithstanding the positive results, Goff emphasized the importance of antibiotic stewardship and continued surveillance in the face of escalating resistance worldwide and the emergence of Klebsiella pneumoniae carbapenemases (KPCs) in some US hospitals.

\section{KPCs in Germany}

Enterobacteriaceae that produce KPCs (which are not exclusive to $K$. pneumoniae) are a particular concern as infections caused by these pathogens are susceptible to very few antibiotics.

European Congress of Clinical Microbiology and Infectious Diseases delegates heard that KPC-producing $K$. pneumoniae have arrived in Germany. In an analysis of imipenem resistance in 49 German ICUs, Meyer and colleagues found seven resistant isolates in 2008 [5]. Two of these were tested and identified as KPC-producing bacteria.

\section{Financial \& competing interests disclosure \\ Attendance and reporting was supported by Schering- Plough. The report was written independently of the company. The author has no other relevant affliations or financial involvement with any organization or entity with a financial interest in or financial conflict with the subject matter or materials discussed in the manuscript apart from those disclosed. \\ No writing assistance was utilized in the production of this manuscript.}

\section{Bibliography}

1. Roede BM, Monen J, van de Sande-Bruinsma N et al.: Rapid increase of antimicrobial-resistant Gram-negative pathogens in Europe. Clin. Microbiol. Inf. 16(Suppl. 2), S27 (2010).

2. Badal R, Bouchillon S, Hoban D et al: Susceptibility of Gram-negative pathogens isolated from intra-abdominal infections in Europe in 2008-2009 - The SMART study. Clin. Microbiol. Inf. 16(Suppl. 2), S354 (2010).
3. Badal R, Bouchillon S, Hoban D et al.: Comparison of antimicrobial susceptibility of Gram-negative pathogens from ICU vs non-ICU - SMART, 2008-2009. Clin. Microbiol. Inf. 16(Suppl. 2), S355 (2010).

4. Goff D, Mangino J: Effect of ertapenem on susceptibility of imipenem to Pseudomonas aeruginosa six years later. Clin. Microbiol. Inf. 16(Suppl. 2), S106 (2010).
5. Meyer E, Schwab F, Schroeren-Boersch B, Gastmeier P: Imipenem-resistant K. pneumoniae and E. coli in 49 German ICUs, 2005-2008. Clin. Microbiol. Inf. 16(Suppl. 2), S363 (2010). 